\title{
A note on the path integral for systems with primary and secondary second class constraints
}

\author{
Marc Henneaux ${ }^{a, b}$ and Andrei Slavnov ${ }^{a, c}$ \\ a Faculté des Sciences, Université Libre de Bruxelles, Campus Plaine C.P. \\ 231, B-1050 Bruxelles, Belgium. \\ ${ }^{b}$ Centro de Estudios Científicos de Santiago, Casilla 16443, Santiago 9, \\ Chile \\ ${ }^{c}$ Steclov Mathematical Institute, Vavilov 42, GSP-1, 117966, Moscow
}

\begin{abstract}
It is shown that the phase space path integral for a system with arbitrary second class constraints (primary, secondary ...) can be rewritten as a configuration space path integral of the exponent of the Lagrangian action with some local measure.
\end{abstract}


The path integral for a system $\left(q^{i}, p_{i}\right)$ with arbitrary second class constraints $\chi_{a}=0$ (primary, secondary ...) and Hamiltonian $H$ is given by

$$
Z=\int \mathcal{D} q \mathcal{D} p \prod_{t}\left|\operatorname{det}\left[\chi_{a}, \chi_{b}\right]\right|^{1 / 2} \delta\left(\chi_{a}\right) \exp i \int\left(p_{i} J \dot{q}^{i}-H\right) d t
$$

(see [1, 2] and [3] for a recent review). The purpose of this note is to show that one can rewrite (1) as the Lagrangian path integral

$$
Z=\int \mathcal{D} q \rho_{L} \exp i J \int L d t
$$

where $L$ is the original Lagrangian and where $\rho_{L}$ is a local measure. Although this result is probably known for some particular cases (free massive vector field), it has not been proved, to our knowledge, in full generality. This is done in this letter.

Our paper is divided in three parts. First, we explain the difficulty in going from (1) to (2). Second, we establish (2). And finally, we comment on the usefulness and applications of the results.

Difficulty : The difficulty in rewriting the Hamiltonian path integral (1) as the Lagrangian path integral (2) stems from the secondary, tertiary ... constraints. Indeed, the path integral (1) is equal to

$$
Z=\int \mathcal{D} q \mathcal{D} p \mathcal{D} \lambda \prod_{t}\left|\operatorname{det}\left[\chi_{a}, \chi_{b}\right]\right|^{1 / 2} \exp i \int\left(p_{i} \dot{q}^{i}-H-\lambda^{a} \chi_{a}\right) d t J,
$$

where the $\delta$-functions of the constraints have been exponentiated by means of Lagrange multipliers. Now, if there were only primary constraints, one could solve the equations $\delta S_{H} / \delta p_{i}=0$ and $\delta S_{H} / \delta \lambda^{a}=0$ for $p_{i}$ and $\lambda^{a}$, which appear thus as auxiliary fields. The value of $S_{H}$ at the extremum for $p_{i}$ and $\lambda^{a}$ is precisely the Lagrangian action $S_{L}$. Therefore, if one evaluates (3) by stationary phase, one gets precisely (2) where the local measure is the product of $\prod_{t}\left|\operatorname{det}\left[\chi_{a}, \chi_{b}\right]\right|^{1 / 2}$ evaluated at the extremum times the contributions coming from the integration over $p_{i}$ and $\lambda^{a}$. The same argument does not apply as such if there are secondary, tertiary, ... constraints since it is then in general impossible to solve the equations $\delta S_{H} / \delta p_{i}=0$ and $\delta S_{H} / \delta \lambda^{a}=0$ for $p_{i}$ and $\lambda^{a}$. Therefore, it is not clear that one can rewrite (1) as a path integral over trajectories in configuration space of the exponential of $i$ times 
the Lagrangian action. Nevertherless, we shall show that this statement is correct by making an appropriate change of variables.

Solution : Let us first consider for definiteness the case of one primary second class constraint $\chi_{1}=0$ and one secondary second class constraint $\chi_{2}=0$. We can always choose the secondary constraint so that

$$
\left[H, \chi_{1}\right]=\chi_{2}
$$

and, of course,

$$
\left[\chi_{1}, \chi_{2}\right] \neq 0 \text {. }
$$

The Hamiltonian path integral (3) is

$$
Z=\int \mathcal{D} q \mathcal{D} p \mathcal{D} \lambda \mathcal{D} \mu \prod_{t}\left|\operatorname{det}\left[\chi_{a}, \chi_{b}\right]\right|^{1 / 2} \exp i \int\left(p_{i} \dot{q}^{i}-H-\lambda \chi_{1}-\mu \chi_{2}\right) d t
$$

We now make the canonical change of variables $\left(q^{i}, p_{i}\right) \rightarrow\left(q^{i}, p_{i}^{\prime}\right)$ generated by $\mu \chi_{1}$,

$$
\begin{gathered}
q^{i} \rightarrow q^{i}=\exp \left(\left[\mu \chi_{1}\right), q^{i}\right] \equiv q^{i}+\left[\mu \chi_{1}, q^{i}\right]+\frac{1}{2}\left[\mu \chi_{1},\left[\mu \chi_{1}, q^{i}\right]\right]+\ldots, \\
p_{i} \rightarrow p_{i}^{\prime}=\exp \left(\left[\mu \chi_{1}\right), p_{i}\right]
\end{gathered}
$$

Under this canonical transformation, the measure, the kinetic term $p \dot{q}$ in the action, and $\chi_{1}$ are invariant. The Hamiltonian becomes

$$
H \rightarrow H^{\prime}=\exp \left(\left[\mu \chi_{1}\right), H\right]=H-\mu \chi_{2}-\frac{1}{2} \mu^{2}\left[\chi_{1}, \chi_{2}\right]+O\left(\mu^{3}\right) .
$$

The secondary constraint transforms also as

$$
\chi_{2} \rightarrow \chi_{2}^{\prime}=\exp \left(\left[\mu \chi_{1}\right), \chi_{2}\right]=\chi_{2}+\mu\left[\chi_{1}, \chi_{2}\right]+O\left(\mu^{3}\right) .
$$

Thus, in terms of the new variables, the path integral reads

$$
\begin{array}{rl}
Z=\int \mathcal{D} q & \mathcal{D} p \mathcal{D} \lambda \mathcal{D} \mu \prod_{t}\left|\operatorname{det}\left[\chi_{a}, \chi_{b}\right]\right|^{1 / 2} \\
& \times \exp i \int\left(p_{i} \dot{q}^{i}-H-\lambda \chi_{1}-\frac{1}{2} \mu^{2}\left[\chi_{1}, \chi_{2}\right]+O\left(\mu^{3}\right)\right) d t
\end{array}
$$


One sees that the term linear in $\mu$ has disappeared from the action. The integration over $\mu$ can be done by stationary phase since the coefficient of $\mu^{2}$ is different from zero. This yields

$$
Z=\int \mathcal{D} q \mathcal{D} p \mathcal{D} \lambda \rho \exp i \int\left(p_{i} \dot{q}^{i}-H-\lambda \chi_{1}\right) d t .
$$

Here, $\rho$ is some local measure. It is given explicitly by

$$
\rho=\prod_{t}\left|\operatorname{det}\left[\chi_{1}, \chi_{2}\right]\right|^{1 / 2}
$$

when the series in $\mu$ in (11) terminates at the second order. In general, there are additional local contributions to the measure (13) which are calculable order by order in the stationary phase expansion. In the path integral (12), only the primary constraint appears. We can thus repeat the derivation given above and integrate over $p_{i}$ and $\lambda$ to reach the desired Lagrangian form.

Obviously, the procedure directly generalizes if there are more than one pair of primary and secondary second class constraints. One can also generalize the derivation to the case when constraints of higher generation (tertiary, quaternary ...) are present. This is done as above, by making a change of variables that eliminates the terms linear in the Lagrange multipliers associated with the secondary, tertiary ... constraints. We shall assume that there is an equal number of constraints at each generation. One can show [4] that the general case can be reduced to this one, in the sense that there may be various chains of generations of different lengths, but that these commute in the Poisson bracket. The Hamiltonian path integral reads then

$$
\begin{aligned}
Z= & \int \mathcal{D} q \mathcal{D} p \mathcal{D} \lambda^{(1)} \mathcal{D} \lambda^{(2)} \ldots \mathcal{D} \lambda^{(L)} \prod_{t}\left|\operatorname{det}\left[\chi_{a}, \chi_{b}\right]\right|^{1 / 2} \\
& \times \exp i \int\left(p_{i} \dot{q}^{i}-H-\lambda^{(1) \alpha} \chi_{\alpha}^{(1)}-\lambda^{(2) \alpha} \chi_{\alpha}^{(2)}-\ldots-\lambda^{(L) \alpha} \chi_{\alpha}^{(L)}\right) d t,
\end{aligned}
$$

where $\chi_{\alpha}^{(1)}$ are the primary constraints, $\chi_{\alpha}^{(2)}$ are the secondary constraints, $\chi_{\alpha}^{(3)}$ are the tertiary constraints ..., chosen so that

$$
\chi_{\alpha}^{(i)}=\left[H, \chi_{\alpha}^{(i-1)}\right], i=2,3, \ldots, L
$$

where $L \geq 2$ is the number of generations. 
The transformation that eliminates the linear terms is a straightforward generalization of $(7),(8)$ and reads explicitly

$$
F(q, p) \rightarrow F^{\prime}=\exp \left(\left[\sum_{k=1}^{L-1} \lambda^{(k+1) \alpha} \chi_{\alpha}^{(k)}\right), F\right] .
$$

The quadratic term generated by the transformation is

$$
\frac{1}{2}\left[\sum_{k=1}^{L-1} \lambda^{(k+1) \alpha} \chi_{\alpha}^{(k)}, \sum_{k=2}^{L} \lambda^{(k) \alpha} \chi_{\alpha}^{(k)}\right]
$$

and is easily verified to be non degenerate using the canonical representation of the brackets given in [4]. One can thus evaluate the integral over $\lambda^{(2) \alpha}$, $\lambda^{(3) \alpha}, \ldots, \lambda^{(L) \alpha}$ by stationary phase, which results in a mere modification in the local measure in the path integral. Then, one can integrate over the momenta $p_{i}$ and the Lagrange multipliers $\lambda^{(1) \alpha}$ of the primary constraints to get the Lagrangian path integral (2).

Comments :J The advantages of the Lagrangian path integral are twofold. First, we avoid having to solve explicitly the higher generation constraints. Second, in the case of a relativistic theory, the exponent in the path integral is manifestly invariant, while secondary or higher generation constraints usually spoil manifest Lorentz invariance.

As we have already discussed, there is in (2) a non trivial local measure which can formally be represented as

$$
\rho_{L}=\exp i \int d t\left(\delta(0) M_{1}+\delta^{2}(0) M_{2}+\ldots\right) .
$$

Of course, this expression is not well defined. To make sense out of it, one should specify some regularization method. If one can use dimensional regularization, all these terms vanish and the Lagrangian local measure $\rho_{L}$ is equal to unity. In that case, we arrive at a very simple and complete expression for the Lagrangian path integral,

$$
Z=\int \mathcal{D} q \exp i \int d t L(q, J \dot{q})
$$

which does not require the knowledge of the Dirac bracket. If, however, dimensional regularization - or some other regularization that makes $\delta(0)$ 
equal to zero - is not available, then one must define the meaning of (18) for the model under consideration.

The form of expression (18) for the local measure should not be taken too literally. In particular, it may not be covariant. However, it does not really mean the breaking of Lorentz invariance because the calculation of the path integral may produce analogous terms compensating the non invariance of the measure. This phenomenon may actually occur already for unconstrained systems. For instance, let us consider the Lagrangian

$$
\mathcal{L}=\frac{1}{2} \partial_{\mu} \phi \partial^{\mu} \phi+\frac{1}{2} g\left(\partial_{\mu} \phi A^{\mu}\right)^{2}
$$

where $A^{\mu}$ is treated as an external field. The canonical quantization of this system leads to the well-known path integral

$$
Z=\int \mathcal{D} \phi \prod_{t}\left|\operatorname{det}\left(1+g\left(A^{0}\right)^{2}\right)\right|^{\frac{1}{2}} \exp i \int \mathcal{L} d x
$$

If taken at face value, this expression is not a Lorentz-invariant functional of $A_{\mu}$. However, careful calculation of the path integral shows that the correlation functions of $\partial_{\mu} \phi(x) \partial_{\nu} \phi(y)$ contains non covariant contributions proportional to $\delta_{\mu 0} \delta_{\nu 0} \delta(x-y)$ which compensate the non covariant contribution from the measure. This is a phenomenon characteristic of theories with derivative couplings.

Another simple model which exhibits the same features and which involves second class constraints is described by the Lagrangian

$$
\mathcal{L}=g \partial_{\mu} \phi A^{\mu}\left(A^{\nu} A_{\nu}\right)+\frac{1}{2} \phi^{2}+\frac{1}{2} J \partial_{\mu} A_{\nu} \partial^{\mu} A^{\nu}
$$

This model has both primary and secondary second class constraints. Application of the previous method leads to the Lagrangian path integral

$$
Z=\int \mathcal{D} \phi \mathcal{D} A \prod_{t}\left|\operatorname{det}\left(\left(A_{\nu} A^{\nu}\right)^{2}+8 A_{0}^{2} A_{\nu} A^{\nu}\right)\right|^{\frac{1}{2}} \exp i \int \mathcal{L} d x
$$

As in the previous example, the local measure is not Lorentz invariant but this non invarianve is again compensated by singular contributions coming from the Lagrangian. 
We close this letter with two additional remarks. First, we considered here only the path integral. A complete definition of the quantum theory necessitates of course the knowledge of the physical states, for which the full constraint spectrum (including the secondary, tertiary ...constraints) and Dirac bracket machinery are relevant. Second, one may easily include first class constraints. This is because first class constraints commute with second class ones. One finds again complete agreement between the Hamiltonian path integral and the Lagrangian path integral obtained by standard Lagrangian tools (e.g. antifield formalism), provided one includes in the latter an appropriate local measure.

\section{Acknowledgements}

This work has been supported in part by a research grant from F.N.R.S., by research contracts with the Commission of the European Community, by ISF-grant MNB000 and by the Russian Fund for Fundamental studies under grant number 94-01-003000a.

\section{References}

[1] L. D. Faddeev, Theor. Math. Phys. 1 (1969) 1.

[2] P. Senjanovic, Ann. Phys. (N.Y.) 100 (1976) 227.

[3] M. Henneaux and C. Teitelboim, Quantization of Gauge Systems, Princeton University Press (Princeton : 1992).

[4] M. Chaichian, D. L. Martinez and L. Lusanna, Ann. Phys. (N.Y.) 232 (1994) 40. 